JOURNAL OF

SYNCHROTRON

RADIATION

ISSN 1600-5775

Received 19 February 2016

Accepted 27 April 2016

Edited by J. F. van der Veen

Keywords: free-electron laser; ultra-largebandwith X-ray pulses; transverse-gradient undulator; simulations.

\section{Generation of ultra-large-bandwidth X-ray free- electron-laser pulses with a transverse-gradient undulator}

\author{
Eduard Prat,* Marco Calvi and Sven Reiche
}

Paul Scherrer Institut, CH-5232 Villigen PSI, Switzerland. *Correspondence e-mail: eduard.prat@psi.ch

A new and simple method to generate X-ray free-electron-laser radiation with unprecedented spectral bandwidth above the $10 \%$ level is presented. The broad bandwidth is achieved by sending a transversely tilted beam through a transverse-gradient undulator. The extent of the bandwidth can easily be controlled by variation of the beam tilt or the undulator gradient. Numerical simulations confirm the validity and feasibility of this method.

\section{Introduction}

X-ray free-electron-laser (XFEL) facilities are leading-edge scientific instruments in multiple research fields like biology, material science, chemistry and physics. XFELs are commonly based on the self-amplified spontaneous emission (SASE) process (Kondratenko \& Saldin, 1980; Bonifacio et al., 1984). SASE-XFELs produce transversely coherent radiation with pulse powers of tens of gigawatts and pulse durations of a few tens of femtoseconds and shorter (Emma et al., 2010). The natural bandwidth of the SASE-XFEL pulses is of the order of the Pierce parameter $\rho$ (Bonifacio et al., 1984), with values between $10^{-3}$ and $10^{-4}$ for a typical XFEL facility. To improve the longitudinal coherence of SASE-XFELs, seeding techniques are employed, which further reduce the radiation bandwidth close to the Fourier limit (Feldhaus et al., 1997; Saldin et al., 2001; Geloni \& Saldin, 2010; Amann et al., 2012).

There is, however, a strong scientific interest in obtaining large-bandwidth XFEL pulses for some applications such as X-ray crystallography (Dejoie et al., 2013; Baradaran et al., 2012; Arthur et al., 2013), X-ray emission and absorption spectroscopy (Patterson et al., 2010), stimulated Raman spectroscopy (Baradaran et al., 2012) and multi-wavelength anomalous diffraction (Son et al., 2011). The generation of broadband XFEL radiation will improve significantly the efficiency of these techniques, paving the way for outstanding progress in research fields such as biology and material science. From the operation point of view, a broad bandwidth allows for more flexibility in the use of the radiation: the XFEL wavelength can be tuned by monochromators without the need to change any parameter on the accelerator side.

The central wavelength $\lambda$ of the XFEL radiation is given by the resonance condition (Bonifacio et al., 1984),

$$
\lambda=\frac{\lambda_{\mathrm{u}}}{2 \gamma^{2}}\left(1+\frac{K^{2}}{2}\right),
$$

where $\lambda_{u}$ is the undulator period length, $\gamma$ is the Lorentz factor of the electron beam and $K$ is the undulator field parameter. A 
natural way to obtain large-bandwidth XFEL pulses is to produce the radiation with an energy-chirped electron beam; i.e. when in the beam there is a correlation between the energy and the longitudinal position of the electrons. For instance, according to equation (1), a $1 \%$ electron energy chirp will generate XFEL pulses with $2 \%$ wavelength bandwidth. Andonian and co-workers obtained radiation pulses at wavelengths in the micrometer range with a total bandwidth of up to $15 \%$ using an electron beam with a central energy of $70 \mathrm{MeV}$ and a large energy chirp (Andonian et al., 2005). In the X-ray regime the electron beam energy needs to be much higher, of the order of gigaelectronvolts. Consequently, obtaining a large energy chirp is much more difficult. Different approaches exist for obtaining the energy chirp at XFEL facilities: optimizing the compression scheme of the linac and using the longitudinal wakefields of the RF structures, as considered in various facilities such as SwissFEL (Saá Hernández et al., 2016) or the Linac Coherent Light Source (Turner et al., 2015); modifying the longitudinal laser profile of the photoinjector (Penco et al., 2014; Saá Herñandez et al., 2016); and utilizing the collective effects of an extremely compressed beam, as proposed for the European XFEL project by Serkez et al. (2013). By using these methods, or combining them, the bandwidth of the XFEL pulses can reach the few-percent level. A possibility to further increase the energy chirp of the beam is given by exploiting the wakefield of dedicated beamline elements such as corrugated pipes and dielectric structures (Craievich, 2010; Emma et al., 2014; Antipov et al., 2014; Zhang et al., 2015). For practical reasons the energy chirp of the electron beam can only be increased up to a few percent, otherwise dispersive and chromatic effects will prevent the amplification of the XFEL process.

\section{Description of the scheme}

We propose a more efficient way to produce large-bandwidth XFEL pulses by using a transverse-gradient undulator (TGU) (Smith et al., 1979; Fuchert et al., 2012) in conjunction with a transversely tilted electron beam. In a TGU there is a linear dependence of the undulator field on the transverse position $x$,

$$
\frac{K(x)-K_{0}}{K_{0}}=\alpha x,
$$

where $K_{0}$ is the on-axis value of the undulator field parameter and $\alpha$ is a constant gradient. In a transversely tilted beam there is a correlation between the transverse and longitudinal positions (time) of the particles. Therefore, if such a beam travels through a TGU it will produce XFEL radiation with broad bandwidth, as shown schematically in Fig. 1. The transverse tilt can be characterized with the tilt amplitudes in offset $\mathrm{d} x / \mathrm{d} s$ and angle $\mathrm{d} x^{\prime} / \mathrm{d} s$, with $x$ being the transverse offset of the electrons, $x^{\prime}$ their transverse angle, and $s$ their longitudinal coordinate.

Figure 1
In our method it is straightforward to tune the bandwidth of the XFEL radiation by modifying the tilt amplitude or the gradient of the TGU or both. This is an additional advantage with respect to previous approaches, which are difficult to set up and to tune (see below for more details).

Increasing the beam size horizontally would also generate broadband radiation with a TGU. However, the increase of the beam size would deteriorate the XFEL performance such that the radiation power would not grow significantly. A transverse tilt overcomes this limitation: with a transverse tilt, although the total beam size is also increased, the transverse size of the volume occupied by the electrons within the cooperation length of the XFEL process (a small fraction of the total bunch length) is almost preserved, therefore the XFEL performance is practically not affected.

In a typical undulator beamline the electrons are focused with quadrupole magnets to have small beam size for an optimum XFEL performance, i.e. maximum XFEL power at a minimum undulator length to achieve saturation. If the beam is transversely tilted, focusing would cause only a small fraction of the beam to produce XFEL radiation, i.e. the part whose betatron oscillations are small enough to allow a good transverse overlap between the electron and photon beams. This concept has been proposed to reduce the XFEL pulse duration (Emma \& Huang, 2004) and to generate high-power and short XFEL pulses (Prat et al., 2015). In our case, however, all the electrons need to produce XFEL radiation and the transverse positions of the electrons must be as constant as possible in order for a longitudinal slice to radiate at the same frequency. Therefore all electrons comprising the beam should travel parallel along the undulator axis. This is achieved if the beam has a transverse tilt only in offset (not in angle) at the undulator entrance and in the absence of external focusing all along the undulator lattice. As a result, the betatron functions within the undulator beamline will be larger than the optimum ones. The electron beam will still see the natural focusing of the undulator. If the betatron phaseadvance along the undulator beamline is much less than $90^{\circ}$, which is normally the case for electron beams with $\mathrm{GeV}$ energies, the change of transverse trajectory due to the undulator focusing can be compensated by tapering the TGU gradient.

In the following we will discuss the implementation of our method and present simulations that demonstrate the feasibility of our scheme. For that we will take as an example the soft X-ray beamline of SwissFEL (Ganter, 2012), which is
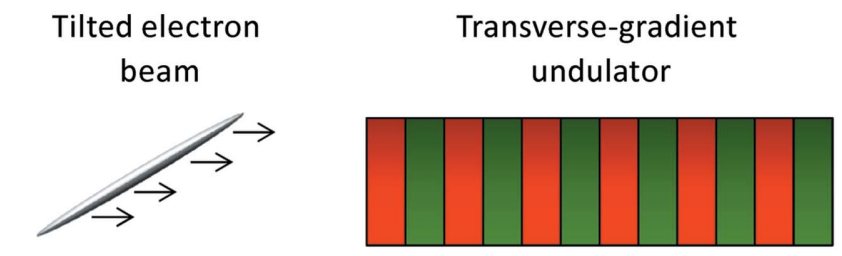
Large-bandwidth XFEL pulse

Sketch to show the working principle of the proposed method: an electron beam with a transverse tilt traveling through a TGU generates large-bandwidth XFEL radiation. 
expected to produce by the year 2020 XFEL radiation in the wavelength range between 0.7 and $7 \mathrm{~nm}$ (photon energies between 0.18 and $1.77 \mathrm{keV}$ ). We note, however, that the method works in general for any photon energy. The undulator line consists of Apple-III devices (Clarke, 2004; Schmidt et al., 2015) with an undulator period of $40 \mathrm{~mm}$. From the wavelength range we select the case of $1 \mathrm{~nm}(1.24 \mathrm{keV})$, which corresponds to a beam energy of $3 \mathrm{GeV}$ and a nominal $K_{0}$ of 1.2.

\subsection{Generation of the TGU gradient}

The gradient can be generated with an undulator with canted poles as proposed by Huang et al. (2012), where a gradient of around $40 \mathrm{~m}^{-1}$ for a standard undulator and of $150 \mathrm{~m}^{-1}$ for a superconducting undulator were considered. A more versatile option consists of employing standard Apple undulators equipped with a variable-gap drive system and four mechanically independent magnetic arrays, as shown in Fig. 2. The gradient can be obtained by shifting longitudinally either the two upper arrays $\varphi_{1}$ and $\varphi_{2}$ against the lower arrays $\varphi_{3}$ and $\varphi_{4}$ (top-bottom shift), or the two left arrays $\varphi_{2}$ and $\varphi_{3}$ against the two right arrays $\varphi_{1}$ and $\varphi_{4}$ (left-right shift). A top-bottom shift generates a gradient in the horizontal direction while a left-right shift produces a gradient in the vertical plane. The sign of the gradient can be inverted by shifting in the opposite direction. With this method the gradient can easily be controlled by varying the longitudinal shifts. Since $K_{0}$ and $\alpha$ are strongly correlated in the case of a fixed-gap device, an undulator with variable gap is required to have independent control over both variables.

The gradient may be determined by measuring the central wavelength of the XFEL radiation with a spectrometer as a function of the transverse offset of the electron beam in the undulator.

In Fig. 3 the operational range of $K_{0}$ and $\alpha$ for the SwissFEL undulators is presented, as computed using the RADIA software package (Chubar et al., 1998). The simulations are calculated assuming samarium-cobalt magnets with a rema-

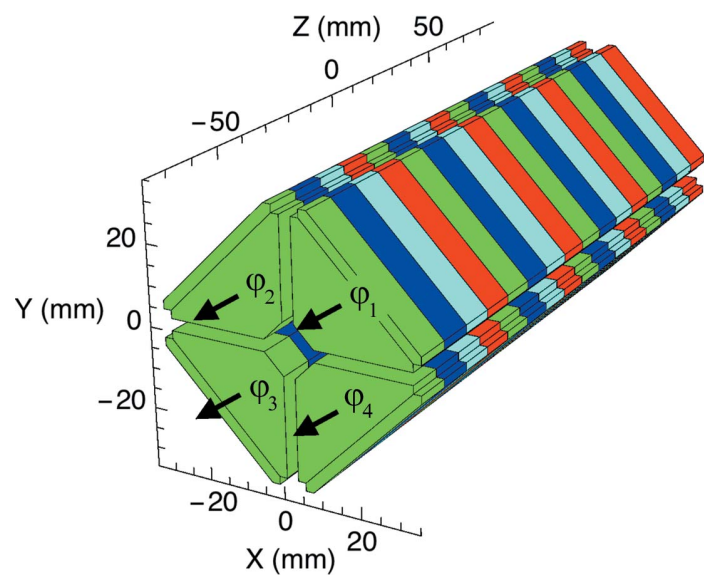

Figure 2

Sketch of the standard Apple undulator device. Each color corresponds to a different magnetization direction and the arrows represent possible longitudinal shifts (with respect to $Z$ ). See text for more details.

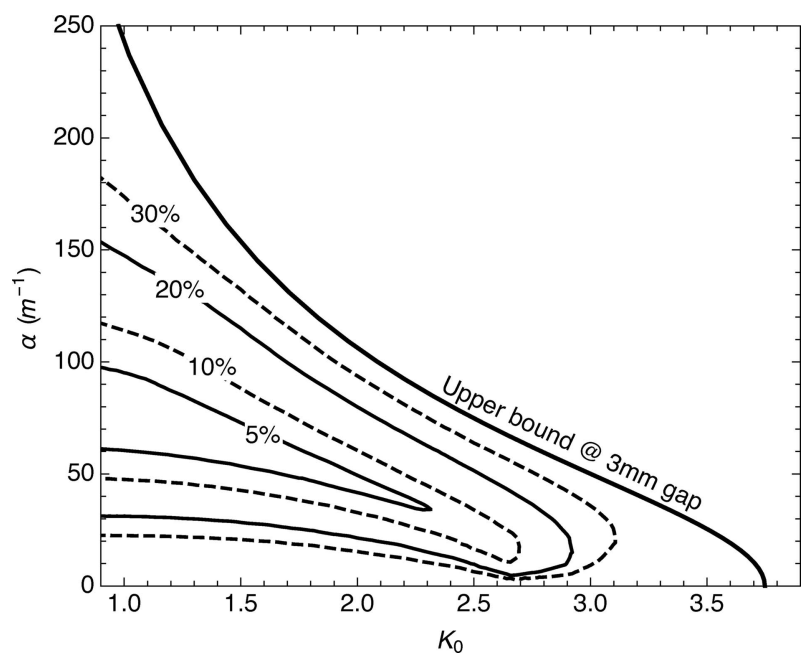

Figure 3

Available gradient for a standard Apple undulator as a function of the undulator field parameter on-axis. The gradient is obtained by shifting longitudinally the arrays of the Apple device. The different lines define areas with a certain level of field linearity, defined as the maximum deviation of the gradient in a region of $\pm 1 \mathrm{~mm}$ with respect to the on-axis gradient. For instance, for $K_{0}=1$, we can obtain a gradient between 30 and $150 \mathrm{~m}^{-1}$ with a linearity better than $20 \%$ (see text for more details).

nence of $1.08 \mathrm{~T}$. The calculations have been performed for circularly polarized light; for linear polarization the obtained gradients are much smaller. The upper bound is calculated with the undulator at its minimum gap $(3 \mathrm{~mm})$ and for topbottom shifts between 0 and $\lambda_{\mathrm{u}} / 2(0$ and $20 \mathrm{~mm})$. The transverse area where the gradient is constant, i.e. where the field is linear, depends on the working point. In Fig. 3 the region of $\pm 1 \mathrm{~mm}$ has been identified and the deviation from constant gradient is estimated as the maximum change of the gradient with respect to its central value. The results are expressed as percentages. As the figure shows, a gradient between $\sim 30 \mathrm{~m}^{-1}$ and $\sim 80 \mathrm{~m}^{-1}$ with a variation smaller than $20 \%$ can be achieved for $K_{0}$ between 1 and 2 .

A TGU with a constant gradient will produce a transverse kick to the beam, which can be easily compensated with a dipole field corrector, and a weak natural focusing (Jha \& Wurtele, 1993). For the gradient considered in our study this focusing strength is 20 times weaker than the natural focusing of a standard undulator, therefore we neglect it in our calculations. For a non-constant gradient this weak natural focusing can be increased significantly. For the simulations presented in this paper, however, the field linearity is good enough to neglect any additional focusing effects.

There are two main limitations in using the standard Apple device. The first concerns the polarization, because this technique works only for circularly polarized light. The second concerns the small range of field linearity when operating with low gradients (below $20 \mathrm{~m}^{-1}$ ). Both these problems can be overcome with the development of a new generation of Apple undulators where a split gap system can independently drive the left and the right magnetic arrays. At SwissFEL the further option of four independent gap drive systems is presently under investigation. 


\subsection{Generation of the transverse tilt}

The transverse tilt of the beam can be generated with different methods, all relying on standard components of XFEL facilities: applying a transverse deflecting RF structure to streak the beam (Loew \& Altenmueller, 1965), introducing dispersion to an energy chirped beam (Prat \& Aiba, 2014), or using the transverse wakefields (Zotter \& Kheifets, 1998) of the accelerating or any other structures of the beamline. The beam tilt can be easily tuned if it is generated with a transverse deflector by simply varying the deflector voltage. If the tilt is created with dispersion, one can modify the dispersion strength and compensate the change in optics and/or trajectory accordingly.

The tilt amplitude can be measured by streaking the beam in the plane perpendicular to the tilt plane; for instance, a horizontal tilt would be measured by streaking the beam in the vertical plane. This diagnostic streaking can be achieved with the same methods as used for the tilt generation.

The transverse deflecting structures foreseen for SwissFEL operate at a C-band frequency $(f=5.72 \mathrm{GHz})$ and provide a total integrated voltage of $70 \mathrm{MV}$ (Craievich et al., 2013). These structures can apply a kick of about $\pm 28 \mu \mathrm{rad}$ to a $3 \mathrm{GeV}$ electron beam with a total length of $20 \mu \mathrm{m}$ (i.e. a duration of $67 \mathrm{fs}$ ). Considering a $\beta$-function at the deflector position of $100 \mathrm{~m}$, this corresponds to a tilt amplitude in offset of 125 at the undulator entrance for our beam optics (i.e. a trajectory offset variation of $\pm 1.25 \mathrm{~mm}$ along the whole bunch length). Stronger tilts can be obtained with the dispersion method, for instance by using a quadrupole magnet in a bunch compression chicane of the facility, where the beam by default has an energy chirp of the order of $1 \%$, as required for the compression process.

\section{Simulations}

According to equations (1) and (2), a beam having a tilt amplitude in offset of 125 traveling through a TGU with a gradient of $48 \mathrm{~m}^{-1}$ will produce radiation with a total bandwidth of $10 \%$. We have performed numerical simulations using the code Genesis 1.3 (Reiche, 1999, 2015) to further investigate this case. The simulated electron beam has the following properties: a flat energy profile with a mean value of $3.0 \mathrm{GeV}$ and a slice energy spread of $350 \mathrm{keV}$, a flat current profile with a value of $3 \mathrm{kA}$ and a total charge of $200 \mathrm{pC}$ (corresponding to a total pulse length of $20 \mu \mathrm{m}$ or $67 \mathrm{fs}$ ), and a normalized transverse emittance of $300 \mathrm{~nm}$. We consider a continuous planar undulator of $40 \mathrm{~m}$ length with natural weak focusing in the vertical plane. Equivalent results in terms of bandwidth would be obtained for a helical undulator; the performance in terms of XFEL power, however, would be better for helical undulators thanks to the higher coupling between the electrons and XFEL radiation. For a total undulator length of $40 \mathrm{~m}$ without external focusing, the matching $\beta$-functions are $40.0 \mathrm{~m}$ and $34.7 \mathrm{~m}$ in the horizontal and vertical planes, respectively. We have run five simulations using different seeds to emulate the shot noise of the electron beam. In all cases the XFEL process reaches saturation within the available undulator length. As an example, Figs. 4 and 5 show the XFEL spectrum and power profile, respectively, at the end of the undulator beamline for one simulation. The total radiation bandwidth is confirmed to be $10 \%$, the total photon pulse duration is $67 \mathrm{fs}$ (i.e. the whole electron bunch is lasing), the XFEL peak power is $8.7 \pm 1.9 \mathrm{GW}$, and the pulse energy at the end of the undulator beamline is $146 \pm 9 \mu \mathrm{J}$, where the error bar indicates the standard deviation of the results over the simulated seeds.

Obviously a given target XFEL bandwidth can be achieved with different values of the TGU gradient and the beam tilt amplitude, but extreme values in either parameter should be avoided. For instance, in the case of a strong tilt the radiation can only slip a small distance before it escapes the electron beam in the forward direction. If this distance, which scales inversely with the tilt amplitude, is shorter than the cooperation length (Bonifacio et al., 1984), the XFEL performance is reduced. Similarly, if the gradient is too large, the wavelength change within the intrinsic transverse beam size of the electrons will also be too large, again harming the XFEL perfor-

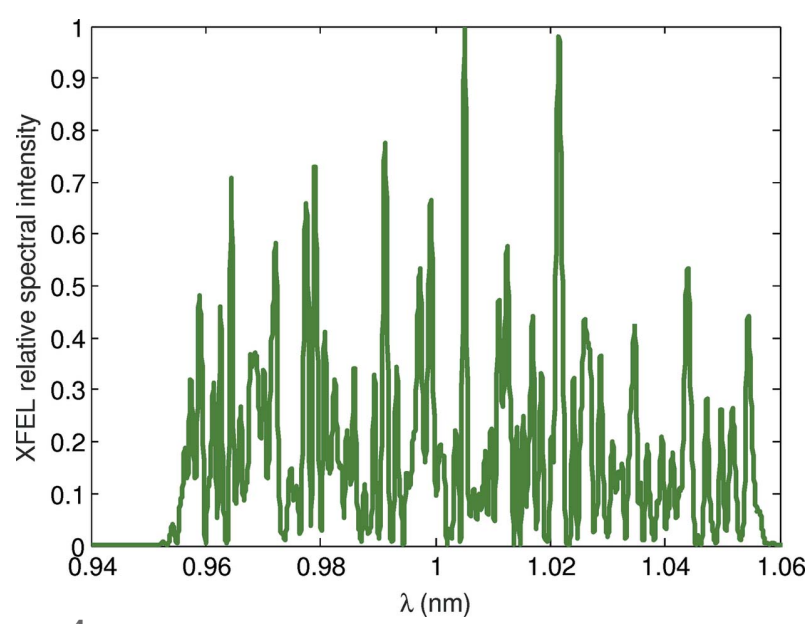

Figure 4

Example of a simulated spectrum for an XFEL pulse with a total bandwidth of $10 \%$. It is obtained with a tilt amplitude in offset of 125 and a TGU gradient of $48 \mathrm{~m}^{-1}$.

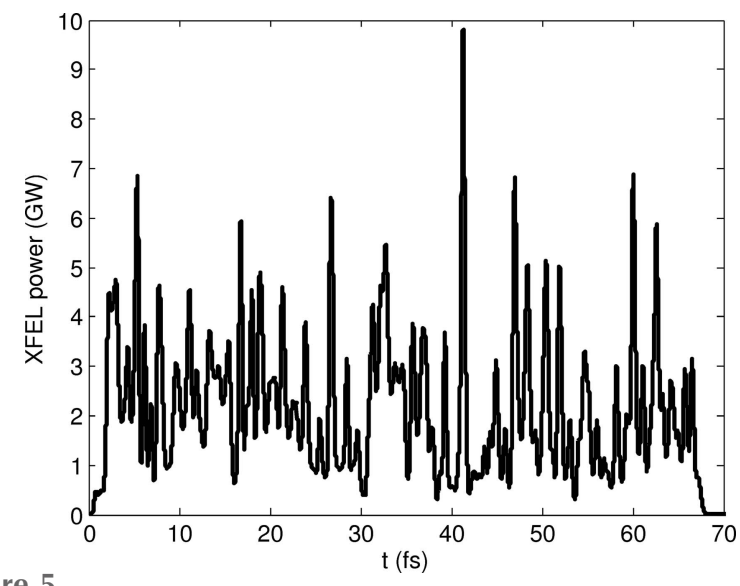

Figure 5

Power profile corresponding to the spectrum shown in Fig. 4. 


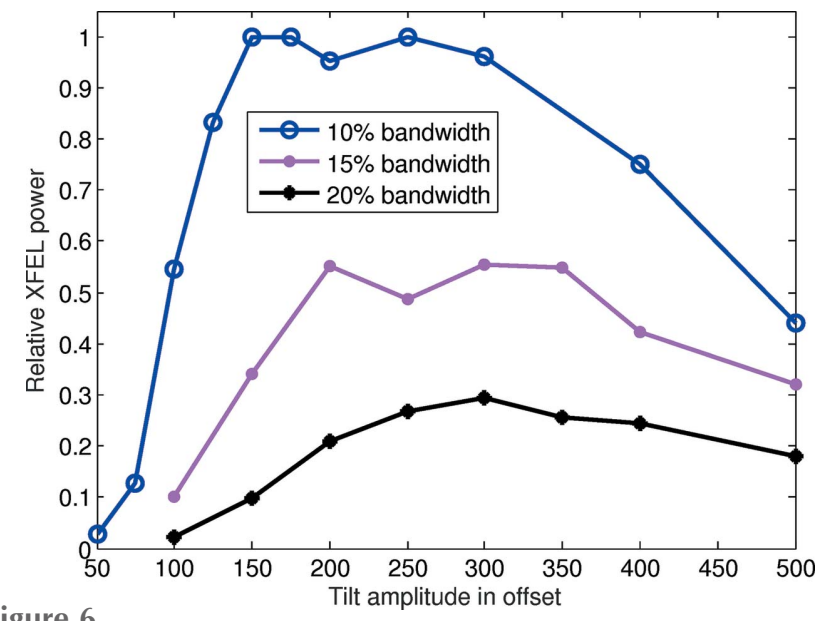

Figure 6

Relative XFEL power as a function of the tilt amplitude to achieve different spectral bandwidths. A relative XFEL power of 1 corresponds to an XFEL pulse energy of $175 \mu \mathrm{J}$. See text for more details.

mance. We have run numerical simulations to identify the optimal configurations for achieving XFEL pulses with $10 \%$, $15 \%$ and $20 \%$ bandwidth. Fig. 6 shows the relative XFEL power as a function of the tilt amplitude in offset for the different bandwidths. For $10 \%$ bandwidth, the optimum performance is found with a tilt amplitude in offset between 150 and 300 , corresponding to a gradient between $40 \mathrm{~m}^{-1}$ and $20 \mathrm{~m}^{-1}$. In our previously simulated case with a tilt amplitude in offset of 125 , the XFEL power is about $20 \%$ below the optimum. As expected, for larger bandwidths (15\% and $20 \%$ ) the achievable XFEL power decreases. The maximum bandwidth that can be realised with our method depends on the minimum power that is required. In our case, we could achieve XFEL pulses with $20 \%$ bandwidth and peak powers at the few-gigawatt level. In general, the optimum gradient/tilt amplitude values will depend on various other parameters, such as, for instance, the central radiation wavelength: for a longer wavelength the cooperation length is larger, thereby reducing the maximum acceptable beam tilt.

\section{Additional possibilities of the scheme}

In our method, short XFEL pulses with different colors can be obtained by simply adding a slotted foil (Emma et al., 2004) in front of the undulator beamline. The foil spoils the transverse beam emittance of the electrons in such a way that only the electrons traveling through the slots can generate XFEL radiation. Therefore, by means of a multiple-slotted foil, multiple short XFEL pulses with different radiation wavelengths can be generated. The pulse duration and the time separation of the XFEL pulses can be controlled by modifying the slot length and separation, respectively, and the wavelength difference between the pulses can be varied with the amplitude of the tilt and the TGU gradient. Multiple-color operation is in high demand by the scientific user community: for instance, the generation of two-color XFEL pulses with a controlled short time delay between them can be used for twocolor pump-probe experiments, which play crucial roles in research fields such as materials science (Baradaran et al., 2012), molecular physics and photochemistry (Ullrich et al., 2012).

The ultra-large-bandwidth XFEL pulses obtained from our method can be compressed using pulse-compression techniques. Such techniques, available for some time in the optical regime, are presently being investigated in view of their efficiency for X-rays (see, for instance, Chapman \& Nugent, 2002; Hrdý \& Oberta, 2013). Considering that the intrinsic bandwidth of the XFEL pulses is of the order of $10^{-3}$ (soft X-rays) and $10^{-4}$ (hard X-rays) and that the pulses obtained from our method have a total bandwidth of $10 \%$ or more, the pulse length could potentially be compressed by a factor of $\sim 100$ (soft X-rays) and $\sim 1000$ (hard X-rays). Consequently, our scheme could in principle be used to obtain multi-terawattattosecond XFEL pulses.

\section{Conclusion}

In summary, we have presented a new and simple method that is able to generate XFEL pulses with ultra-large-bandwidth above the $10 \%$ level. The large bandwidth is achieved by sending a transversely tilted beam through a TGU. In this scheme the bandwidth can easily be tuned by modifying the tilt amplitude and/or the gradient of the TGU. Our numerical simulations demonstrate the validity of the method for the SwissFEL case. The novel technique opens up new opportunities for experiments requiring broad-bandwidth XFEL radiation or XFEL pulse trains with multiple colors.

\section{Acknowledgements}

We would like to acknowledge Hans Braun and Thomas Schietinger for productive discussions and meticulous proofreading of this document.

\section{References}

Amann, J. et al. (2012). Nat. Photon. 6, 693-698.

Andonian, G., Murokh, A., Rosenzweig, J. B., Agustsson, R., Babzien, M., Ben-Zvi, I., Frigola, P., Huang, J. Y., Palumbo, L., Pellegrini, C., Reiche, S., Travish, G., Vicario, C. \& Yakimenko, V. (2005). Phys. Rev. Lett. 95, 054801.

Antipov, S., Baturin, S., Jing, C., Fedurin, M., Kanareykin, A., Swinson, C., Schoessow, P., Gai, W. \& Zholents, A. (2014). Phys. Rev. Lett. 112, 114801.

Arthur, J. et al. (2013). LCLS-II new instruments workshops report. Report R-1006. SLAC National Accelerator Laboratory, USA.

Baradaran, S. et al. (2012). LCLS-II new instruments workshops report. Report R-993. SLAC National Accelerator Laboratory, USA.

Bonifacio, R., Pellegrini, C. \& Narducci, L. M. (1984). Opt. Commun. 50, 373-378.

Chapman, H. N. \& Nugent, K. A. (2002). Opt. Commun. 205, 351-359.

Chubar, O., Elleaume, P. \& Chavanne, J. (1998). J. Synchrotron Rad. 5, 481-484.

Clarke, J. A. (2004). The Science and Technology of Undulators and Wigglers. Oxford University Press.

Craievich, P. (2010). Phys. Rev. ST Accel. Beams, 13, 034401.

Craievich, P., Ischebeck, R., Loehl, F., Orlandi, G. L. \& Prat, E. (2013). Proceedings of the 35th International Free-Electron Laser Conference, pp. 236-241. 
Dejoie, C., McCusker, L. B., Baerlocher, C., Abela, R., Patterson, B. D., Kunz, M. \& Tamura, N. (2013). J. Appl. Cryst. 46, 791-794. Emma, P. et al. (2010). Nat. Photon. 4, 641-647.

Emma, P., Bane, K., Cornacchia, M., Huang, Z., Schlarb, H., Stupakov, G. \& Walz, D. (2004). Phys. Rev. Lett. 92, 074801.

Emma, P. \& Huang, Z. (2004). Nucl. Instrum. Methods Phys. Res. A, 528, 458-462.

Emma, P., Venturini, M., Bane, K. L. F., Stupakov, G., Kang, H.-S., Chae, M. S., Hong, J., Min, C.-K., Yang, H., Ha, T., Lee, W. W., Park, C. D., Park, S. J. \& Ko, I. S. (2014). Phys. Rev. Lett. 112, 034801.

Feldhaus, J., Saldin, E. L., Schneider, J. R., Schneidmiller, E. A. \& Yurkov, M. V. (1997). Opt. Commun. 140, 341-352.

Fuchert, G., Bernhard, A., Ehlers, S., Peiffer, P., Rossmanith, R. \& Baumbach, T. (2012). Nucl. Instrum. Methods Phys. Res.A, 672, 33-37.

Ganter, R. (2012). SwissFEL conceptual design report. Report 10-04. Paul Scherrer Institute, Switzerland.

Geloni, G. K. V. \& Saldin, E. (2010). A simple method for controlling the line width of SASE X-ray FELs. Report 10-053. Deutsches Elektronen-Synchrotron, Hamburg, Germany.

Hrdý, J. \& Oberta, P. (2013). J. Synchrotron Rad. 20, 550-554.

Huang, Z., Ding, Y. \& Schroeder, C. B. (2012). Phys. Rev. Lett. 109, 204801.

Jha, P. \& Wurtele, J. S. (1993). Nucl. Instrum. Methods Phys. Res. A, 331, 477-481.

Kondratenko, A. M. \& Saldin, E. L. (1980). Part. Accel. 10, 207216.

Loew, G. A. \& Altenmueller, O. H. (1965). Design and applications of $R F$ deflecting structures at SLAC. Report PUB-135. SLAC National Accelerator Laboratory, SLAC, USA.

Patterson, B. D., Abela, R., Braun, H. H., Flechsig, U., Ganter, R., Kim, Y., Kirk, E., Oppelt, A., Pedrozzi, M., Reiche, S., Rivkin, L., Schmidt, T., Schmitt, B., Strocov, V. N., Tsujino, S. \& Wrulich, A. F. (2010). New J. Phys. 12, 035012.
Penco, G., Danailov, M., Demidovich, A., Allaria, E., De Ninno, G., Di Mitri, S., Fawley, W. M., Ferrari, E., Giannessi, L. \& Trovó, M. (2014). Phys. Rev. Lett. 112, 044801.

Prat, E. \& Aiba, M. (2014). Phys. Rev. ST Accel. Beams, 17, 032801.

Prat, E., Löhl, F. \& Reiche, S. (2015). Phys. Rev. ST Accel. Beams, 18, 100701.

Reiche, S. (1999). Nucl. Instrum. Methods Phys. Res. A, 429, 243-248.

Reiche, S. (2015). Proceedings of the 36th International Free-Electron Laser Conference, pp. 403-407.

Saá Hernández, A., Prat, E., Bettoni, S., Beutner, B. \& Reiche, S. (2016). Submitted.

Saldin, E. L., Schneidmiller, E. A., Shvyd'ko, Y. V. \& Yurkov, M. V. (2001). Nucl. Instrum. Methods Phys. Res. A, 475, 357-362.

Schmidt, T., Anghel, A., Böhler, P., Brügger, M., Calvi, M., Danner, S., Huber, P., Keller, A. \& Locher, M. (2015). Proceedings of the 36th International Free-Electron Laser Conference, pp. 116-120.

Serkez, S., Kocharyan, V., Saldin, E., Zagorodnov, I., Geloni, G. \& Yefanov, O. (2013). Extension of SASE bandwidth up to $2 \%$ as a way to increase the efficiency of protein structure determination by X-ray nanocrystallography at the European XFEL. Report 13-109. Deutsches Elektronen-Synchrotron, Hamburg, Germany.

Smith, T. I., Madey, J. M. J., Elias, L. R. \& Deacon, D. A. G. (1979). J. Appl. Phys. 50, 4580-4583.

Son, S.-K., Chapman, H. N. \& Santra, R. (2011). Phys. Rev. Lett. 107, 218102.

Turner, J. L. et al. (2015). Proceedings of the 36th International FreeElectron Laser Conference, pp. 337-341.

Ullrich, J., Rudenko, A. \& Moshammer, R. (2012). Annu. Rev. Phys. Chem. 63, 635-660.

Zhang, Z., Bane, K., Ding, Y., Huang, Z., Iverson, R., Maxwell, T., Stupakov, G. \& Wang, L. (2015). Phys. Rev. ST Accel. Beams, 18, 010702.

Zotter, B. W. \& Kheifets, S. (1998). Impedances and Wakes in High Energy Particle Accelerators. Singapore: World Scientific. 